\title{
XI. Ueber die Empfindlichkeit von Collodien bei verschiedenem Gehalt an Pyroxylin und Jodirungssalzen; von Emil Zettnov.
}

Soviel mir bekannt, ist Hr. Dr. Vogel (Photogr. Mitth. 1871 Aprilheft) der einzige, welcher einige Versuche darüber angestellt hat, wie stark man ein bestimmtes Rohcollodium jodiren darf; er fand als bestes Verhältnil's für das verwendete circa $1_{4}^{\frac{s}{4}}$ Proc. Jodirungssalze entsprechend $1 \frac{1}{4}$ Proc. Jod und Brom. Um nun nicht nur den Einflufs, welchen ein steigender Gehalt an Jodirungssalzen, sondern auch an Pyroxylin hervorbringt, kennen zu lernen, setzte ich 42 verschiedene Collodien an, welche, wie aus der unten folgenden Tabelle ersichtlich ist, 7 verschiedene Reihen à 6 Stück bildeten, so dafs bei demselben Gehalt an Jodirungssalzen der Gehalt an Pyroxylin von $\frac{1}{4}$ bis $1 \frac{1}{2}$ Proc. schwankte. Vorläufige Versuche hatten ergeben, dafs ein Collodium von 1 Proc. Gehalt an Pyroxylin 5 Proc. Jod und Brom entsprechend circa 7 Proc. Salzen, aufnehmen kann, ohne dafs beim Silbern die Schicht Jodund Bromsilber fahren lälst.

Die Collodien wurden innerhalb zwei Tagen folgendermafsen angesetzt:

Das zur Darstellung verwendete Collodiumpapier war lufttrocken, wurde in kleine Stücke zerschnitten und von demselben innerhalb $1 \frac{1}{4}$ Stunde sämmtliche 42 Proben abgewogen und in die bereit stehenden Flaschen gethan. Vorversuche hatten gezeigt, dafs ein Collodium von $1 \frac{1}{2}$ Proc. Gehalt an Papier sich auf Visitenplatten noch giefsen liefs, während es bei $1 \frac{3}{4}$ Proc. nicht mehr verarbeitet werden konnte.

Alsdann wurde von ein und demselben Alkohol bei $17^{\circ}$ bis $18^{\circ} \mathrm{C}$. die berechnete Menge aus einer in $\frac{1}{5} \mathrm{CC}$. getheilten Bürette abgelassen; hieranf der Aether und schliefslich die Jodirung in derselben Art hinzugefügt und 
die Collodien bis zur Lösung des Papiers, welches einen kaum bemerkbaren Satz hinterliefs, tüchtig geschüttelt.

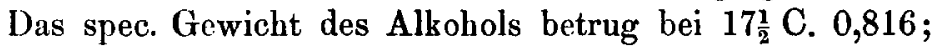
dasjenige des Aethers 0,72.5. Die Jodirung, welche keine Kadminmsalze enthielt, wog 1,036; dieselbe enthielt 20Proc. Jod und Brom entsprechend 27 bis 28 Proc. festen Salzen und kam in derselben auf 3 Theile Jod, 1 Theil Brom. Dals das Abmessen statt des Abwägens hinreichende Genauigkeit gewährte, hatten 6 Controllversuche gelehrt; bei denselben wogen die abgemessenen Quantitäten statt $100 ; 0$

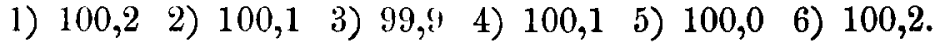
In allen Collodien verhielt sich die Menge des Alkohols zu der des Aethers wie $2: 1$.

\section{$\mathbf{T}_{\text {il belle. }}$}

I. Reihe. Collodien mit $\frac{1}{2}$ Proc. $\mathrm{J}+\mathrm{Br}$.

1.

0,25 Papier.

2,5 Jodirung $\frac{1,8}{64,3}=2,3 \mathrm{CC}$.

\begin{aligned} & 64,3 Alk $=78,8 \% \\ & 33,0$ Aeth $=45,2 \% \\ &$\hline 100,$0 ;{ }_{4}^{1}$ Proc. $\mathrm{I}_{2} ; \frac{1}{2}$ Proc. $\mathrm{J}+\mathrm{Br}\end{aligned}$.

2.

0,5 Papier.

2,5 Jodirung $\frac{1,8}{64,1}=2,8 \mathrm{CC}$.

64,1 Alk $=78,6$ \%

32,9 Aeth $=75,1$,

$\overline{100,0 ; \frac{1}{2}}$ Proc. ${ }^{\prime} ; \frac{1}{2}$ Proc. J + Br.

3.

0,75 Papier.

2,5 Jodirung $\frac{1,8}{64,0}=2,3 \mathrm{CC}$.

64,0 Alk $=78,2$,

32,8 Aeth $=44,9$ "

100,$0 ;{ }_{1}^{3}$ Proc. P ; $\frac{1}{2}$ Proc. J + Br. 
1,0 Papier.

2,5 Jodirung $\frac{62,8}{65,6}=2,3 \mathrm{CC}$.

\begin{tabular}{rlr}
63,8 & Alk $=78,2$ \\
32,7 & Aeth & $=44,8$ \\
\hline 100,$0 ; 1$ & Proc. P; $\frac{1}{2}$ Proc. J + Br.
\end{tabular}

5.

1,25 Papier.

2,5 Jodirung $\frac{1,8}{65,0}=2,3$ CC.

63,6 Alk $=78,0$ \%

32,7 Aeth $=44,8$,

100,$0 ; 1 \frac{1}{4}$ Proc. P; $\frac{1}{2}$ Proc. J + Br.

1,5 Papier.

6.

2,5 Jodirung $\frac{1,8}{65,4}=2,3 \mathrm{CC}$.

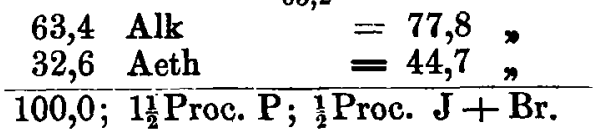

II. Reihe. Collodíen mit 1 Proc. $\mathbf{J}+\mathbf{B r}$.

7.

0,25 Papier.

5,0 Jodirung $\frac{3,5}{62,1}=4,8 \mathrm{CC}$.

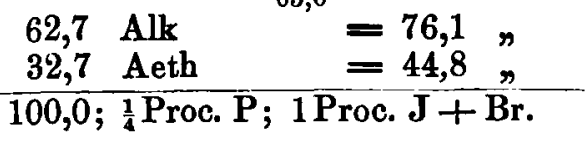

8.

0,5 Papier.

5,0 Jodirung $\frac{3,5}{65,8}=4,8 \mathrm{CC}$.

61,8 Alk $=75,7$,

$\frac{32,7 \text { Aeth }}{=44,8 \text { त }}$ 
9.

0,75 Papier.

5,0 Jodirung $\begin{array}{r}3,5 \\ 61,7\end{array}=4,8 \mathrm{CC}$.

61,7 Alk ${ }^{65,2}=75,3$ n

32,6 Aeth $=44,5 \pi$ 100,$0 ; \frac{3}{4}$ Proc. P; 1 Proc. J + Br.

1,0 Papier.

10.

5,0 Jodirung $\frac{3,5}{61,5}=4,8 \mathrm{CC}$.

61,5 Alk $=75,3$ ” 32,5 Aeth $=44,5$, $\overline{100,0 ;} \overline{1}$ Proc. $\mathbf{P} ; 1$ Proc. $\mathrm{J}+\overline{\mathrm{Br}}$.

\section{1.}

1,25 Papier.

5,0 Jodirung $\frac{3,5}{64,3}=4,8$ CC.

61,3 Alk $=75,1$,

32,4 Aeth $=44,4$ 100,$0 ; 1 \frac{1}{4}$ Proc. 1 ; 1 Proc. $\mathrm{J}+\mathrm{Br}$.

1,5 Papier.

12.

5,0 Jodirung $\frac{3,5}{61,2}=4,8 \mathrm{CC}$.

61,2 Alk $=75,0$, 32,3 Aeth $=44,3$, 100,$0 ; 1 \frac{1}{2}$ Proc. $\mathbf{P} ; 1$ Proc. $\mathrm{J}+\overline{\mathrm{Br}}$.

III. Reihe. Collodien mit $1 \frac{1}{2}$ Proc. $\mathrm{J}+\mathrm{Br}$.

13.

0,25 Papier.

7,5 Jodirung $\frac{5,3}{6,7}=7,2$ CC.

59,7 Alk $=73,2$,

32,6 Aeth

$=44,7$

100,$0 ; \frac{1}{4}$ Proc. $\mathrm{P} ; 1 . \overline{1}$ Proc. $\mathrm{J}+\mathrm{Br}$ 
0,5 Papier.

7,5 Jodirung $\frac{59,3}{64,8}=7,2$ CC.

\begin{aligned} 59,5 Alk & $=73,0 \% \\ 32,5$ Aeth & $=44,5 \% \\$\hline 100,$0 ; & \frac{1}{2}$ Proc. P; $1 \frac{1}{2}$ Proc. J $+\overline{B r}\end{aligned}$.

15.

0,75 Papier.

7,5 Jodirung $\frac{59,4}{6,4}=7,2$ CC.

59,4 Alk $=72,8$ \%

$\begin{aligned} 32,4 & \text { Aeth } \\ 100,0 ; & \frac{3}{4} \text { Proc. P; }\end{aligned}$

1,0 Papier.

16.

7,5 Jodirung $\frac{59,3}{64,5}=7,2$ CC.

59,4 Alk $=72,5$,

$\frac{32,3 \text { Aeth }}{100,0 ; \text { 1 Proc. P; } 1 \frac{1}{2} \text { Proc. } \mathrm{J}+\mathrm{Br} \text {. }}$

1,25 Papier.

17.

7,5 Jodirung $\frac{5,3}{64,1}=7,2$ CC.

58,1 Alk $=72,4$,

32,1 Aeth $=44,1$,

100,$0 ; 1 \frac{1}{4}$ Proc. P; $1 \frac{1}{2}$ Proc. J + Br.

1,5 Papier.

18.

7,5 Jodirung $\frac{5,3}{58,9}=7,2 \mathrm{CC}$.

58,9 Alk $=72,2$,

32,1 Aeth $=44,0$,

100,$0 ; 1 \frac{1}{2}$ Proc. $\mathrm{P} ; 1 \frac{1}{2}$ Proc. J $+\mathrm{Br}$. 
IV. Reihe. Collodien mit 2 Proc. $\mathbf{J}+\mathrm{Br}$.

19.

0,25 Papier.

10,0 Jodirung $\frac{77,2}{64,7}=9,7$ CC.

57,5 Alk

$=70,5$,

$\frac{32,3 \text { Aeth }}{100,0 ;{ }_{4}^{1} \text { Proc. P; } 2 \text { Proc. } J+\overline{B r} \text {. }}$

20.

0,5 Papier.

10,0 Jodirung $\frac{7,27,3}{65,4}=9,7$ CC.

57,5 Alk $=70,3$ \%

32,3 Aeth $=44,1$ n

21.

0,75 Papier.

10,0 Jodirung $\frac{7,2}{64,3}=9,7$ CC.

57,1 Alk $=70,0$,

32,2 Aeth $=44,1$,

100,0; $\frac{3}{4}$ Proc. I'; 2 Proc. J + Br.

22.

1,0 Papier.

10,0 Jodirung $\frac{7,2}{64,9}=9,7$ CC.

50,9 Alk $=69,8$ 力

$\frac{32,1 \text { Aeth }}{100,0 ; 1 \text { Proe. }} \frac{=44,0 \%}{\text { P; 2Proc. J + Br. }}$

1,25 Papier.

23.

10,0 Jodirung $\frac{7,2}{66,7}=9,7$ CC.

56,7 Alk $=69,5$ n

$\frac{32,0 \text { Aeth }}{100,0 ; 1 \frac{1}{4} \text { Proc. P; } 2 \text { Proc. } J+B \text { Br. }}$ 


\section{4.}

1,5 Papier.

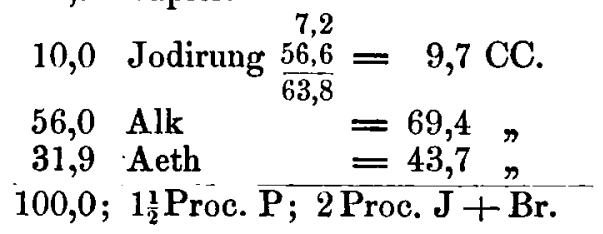

V. Reihe. Collodien mit 3 Proc. J + Br.

Dieselbe umfafste die No. 25 bis 30. Da diese 4 Proc. Collodien ebenso wie die der Reihe VI und VII mit 5 Proc. $\mathrm{J}+\mathrm{Br}$ keine brauchbaren Resultate ergab, so übergehe ich ihre genauere Zusammensetzung und führe nur die aus diesen Reihen geprüften No. 25, 35, 40 an.

25.

0,25 Papier.

\begin{tabular}{rlr}
15,0 & Jodirung & $\frac{10,8}{52,0}=14,5 \mathrm{CC}$. \\
52,9 & Alk $\quad=64,9$ \\
31,9 Aeth & $=43,7$ \\
\hline 100,$0 ; \frac{1}{4}$ Proc. P; & 3 Proc. J + Br.
\end{tabular}

1,25 Papier.

35.

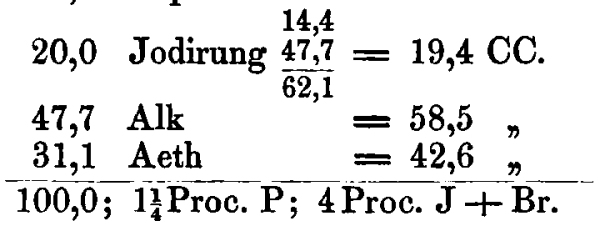

1,0 Papier.

40.

25,0 Jodirung $\frac{17,9}{81,3}=24,2$ CC.

\begin{tabular}{rlr}
43,4 Alk & $=53,2$ \\
30,6 & Aeth & $=41,9$ \\
\hline 100,$0 ;$ & 1Proc. P; 5 Proc. J + Br.
\end{tabular}


Ungefähr drei Wochen nach dem Ansetzen der Collodien wurden dieselben geprüft. (Diese Versuche wurden mit freundlicher Erlaubnifs des Hrn. Dr. Vogel in dem photographischen Atelier der Königl. Gewerbe-Akademie ausgeführt, wozu ich Betreffendem zu tiefstem Dank verpflichtet bin.) Mit Ausnahme von 4 Stück waren sie sämmtlich farblos geblieben und wurden mit einigen Tropfen einer Auflösung von Jod in Alkohol versetzt, so dafs sie weingelb erschienen. Sie arbeiteten alsdann schleierfrei, während sie sonst bedeutend unempfindlicher waren und schleierige Bilder zeigten.

Die Prüfung geschah an wolkenlosen Tagen Ende Dezember und Anfang Januar, im Durchschnitt von 11 bis $1 \frac{1}{2}$ Uhr. Von jedem Collodium wurden zwei Platten gemacht in dieser Reihenfolge: 1 und 2;2 und 3; 3 und 4; 4 und 5 usw. Das von der Platte ablaufende Collodium wurden in einer Abgulsflasche aufgefangen; da sonst bei der zweiten Platte nicht dasselbe Collodium in Anwendung gekommen wäre, wie bei der ersten. Die Platten waren mit Eiweifs überzogen; das Silberbad blieb während der Versuche dasselbe und es wurde in zwei Schalen gesilbert. Die Entwickelung geschah mit dem gewöhnlichen, mit etwas Schwefelsäure angesäuırten Entwickeler; die Fixirung mit unterschwefligem Natron. Als Object bei den Aufnahmen diente eine Gypsbüste mit schwarzer Draperie.

$\mathrm{Zu}$ dünn beim Giefsen erwiesen sich sämmtliche Collodien mit ${ }_{4}^{1}$ und $\frac{1}{2}$ Proc. Papiergehalt; gut liefsen sich die Collodien mit $\frac{3}{4}$ und 1 resp. für kleine Platten $1 \frac{1}{4}$ Proc. giefsen, während die Collodien mit $1 \frac{1}{2}$ Proc. Papiergehalt sich viel zu dick erweisen und sehr leicht Gufsstreifen zeigten.

Als Resultate nach dem Vergleichen der Bilder ergeben sich folgende:

1) Bei demselben Gehalte an Pyroxylin bedingt die Vermehrung der Jodirungssalze bis zu einem gewissen Punkte eine gröfsere Empfindlichkeit, während sie bei weiterer Steigerung nicht nur abnimmt, sondern auch die Bilder 
unklarer und verschwommener werden. Die Collodien 1; $7 ; 13 ; 19 ; 25$ enthielten je $\frac{1}{4}$ Proc. Pyroxylin und $\frac{1}{2} ; 1$; $1 \frac{1}{2}$; 2; 3 Proc. J+Br. Das relativ beste Bild lieferte 13, während bei 7 und 19 das Bild stärker war, als bei 1; unbrauchbar zeigte sich 25, da die Schicht beim $W$ aschen unter starkem Strahle sich stellenweise ablöste. Aehnlich verhielten sich die Nummern 2;8;14;20;26 mit je $\frac{1}{2}$ Proc. Pyroxylin und $3 ; 9 ; 15 ; 21 ; 27$ mit je ${ }_{4}^{3}$ Proc. Die besten Bilder lieferten die Nummern 14 und 15, während 26 und 27 verschwommen und unklare Bilder gaben, welchen diejenigen von No. 3;8; 9 vorzuziehen waren.

2) Die Empfindlichkeit und Schönheit des Bildes steigt mit dem Gehalt an Pyroxylin und zwar so bedeutend, dafs diese durchans nicht der dickeren Jod- und Bromsilberschicht zugeschrieben werden, welche sich in Folge der dickeren Consistenz des Collodiums bildet. Als Beispiel führe ich folgende Bilder an: No. 6 ( $1 \frac{1}{2}$ Proc. Pyroxylin, $\frac{1}{2}$ Proc. $J+B r$.) gab nach dem Silbern eine Schicht, welche die meiste Aehnlichkeit mit No. 7 ( $\frac{1}{4}$ Proc. Pyroxylin, 1 Proc. $J+B r$.) hatte; eher war die letztere undurchsichtiger. Die erhaltenen Bilder waren jedoch aufserordentlich verschieden. Während Bild No. 6 in den Schwärzen mit 20 Sekunden sich völlig ausexponirt erwies, zeigte No. 7 selbst mit 30 Sekunden keine genügenden Details in denselben, fermer war der Gyps in 7 nicht so brillant als in 6 . Beide Platten waren dicht hinter einander geprüft. No. 6 ähnelte am meisten No. 11. Aehnliches ergab sich beim Betrachten der Bilder von No. 12 und 13. Gleich starke und gut entwickelte Bilder zeigten No. 2 und 7; ferner 3 und 13; während 19 ebenso stark als 3 und 13, zeigte es sich jedoch nicht so brillant als diese. Es scheint fast, als ob in diesen Fällen ein gewisser Antheil Pyroxylin ( $\frac{1}{4}$ Proc.) dieselbe Wirkung ausübt wie ein grölserer Antheil an Jodirungssalzen ( $\frac{1}{2}$ Proc.).

3) Sämmtliche Collodien mit $\frac{1}{4}$ bis $\frac{1}{2}$ Proc. Papiergehalt arbeiten flauer als die mit gröfserem Papiergehalt.

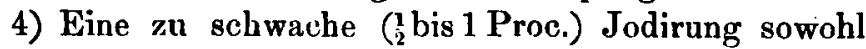


als eine zu starke (bis 5 Proc.) liefert leicht Bilder, welche nicht so harmonisch ausyebildet, und in welchen die Lichter sowohl als die Schatten nicht so klar und detaillirt gezeichnet sind, wie bei nittlerem Gehalt an Jodirung $1 \frac{1}{2}$ bis 2 Proc.).

5) Die vorzüglichsten Bilder bei einer Exposition von 10 bis 12 Sekunden im Januar lieferten die Collodien 15; 16; 17; die gesilberte Schicht ist bei denselben nur schwach durchscheinend. Diesen schlossen sich an No. 10 und 11 mit 20 Sekunden Exposition.

6) Die Collodien 19 bis 24 mit 3 Proc. Jod und Brom entsprechend circa 4 Proc. Salzen, lieferten verschwommene Bilder und hei No. 19 wurden beim Waschen unter starkem Wasserstrahl einige Stellen fortgespült. Von No. 25 ab zeigten siıh die Collodien untauglich; folgende herausgegriffene Nummern, 25, 35 und 40, zeigten zwar eine nach dem Silbern gleichmälsige Schicht, welche jedoch unempfindlich war und beim Entwickeln nur ein schwaches Bild lieferte. Beim Fixiren verschwand das letztere entweder völlig oder es blieben nur schwache Conturen desselben stehen; das unter dem Bilde liegende Jod- und Bromsilber schwemmte beim Lösen den gesammten Silberniederschlag mit fort. Gelbstreifige Platten habe ich niemals erhalten.

Als praktisches Resultat würde sich wohl nicht nur für das oben verwendete, sondern auch für andere Collodien folgendes ergeben: Man setzt das Rohcollodium so dick wie möglich an und jodirt dasselbe so stark, dafs sich in dem fertigen Jod-Collodium $1 \frac{1}{2}$ bis $1 \frac{3}{4}$ Proc. Jod und Brom, resp. 2 bis $2 \frac{1}{2}$ Proc. Salze befinden.

Berlin, 25. Januar 1872. 